\title{
Safety of bloodless autologous stem cell transplantation in Jehovah's Witness patients
}

\author{
Alyssa Beck ${ }^{1} \cdot$ Robert Lin ${ }^{1} \cdot$ Ali Reza Rejali ${ }^{2} \cdot$ Muni Rubens $^{3} \cdot$ Ronald Paquette $^{4} \cdot$ Robert Vescio $^{4}$ Noah Merin ${ }^{4}$. \\ Margarita Guerrero ${ }^{4}$ - Yvette Federizo ${ }^{4}$. Michelle Lua ${ }^{4}$. Leticia Uy ${ }^{4}$. Lorraine Hernandez ${ }^{4}$. Mohana Allred ${ }^{4}$. \\ Ronald Legaspi ${ }^{4} \cdot$ Melissa Leaverton $^{4} \cdot$ Sara Oliva $^{4} \cdot$ Rhona Castillo $^{4} \cdot$ Lorna Dean $^{4} \cdot$ Jennifer Bourke ${ }^{4} \cdot$ Sara Cooper $^{4}$. \\ Seda Gharapetian ${ }^{4} \cdot$ Jose Causin $^{4} \cdot$ Christopher Lopiccolo $^{4} \cdot$ Laura Ann Snoussi ${ }^{4} \cdot$ Patricia VanStrien $^{4} \cdot$ Michael Lill $^{4}$. \\ Yuliya P. Linhares ${ }^{4}$
}

Received: 10 June 2019 / Revised: 26 November 2019 / Accepted: 10 December 2019 / Published online: 2 January 2020

(c) The Author(s) 2020. This article is published with open access

\begin{abstract}
Due to the curative potential and improvement in progression-free survival (PFS), high-dose chemotherapy followed by autologous stem cell transplantation (ASCT) is considered the standard of care for several hematologic malignancies, such as multiple myeloma, and lymphomas. ASCT typically involves support with blood product transfusion. Thus, difficulties arise when Jehovah's Witness patients refuse blood transfusions. In order to demonstrate the safety of performing "bloodless" ASCT (BL-ASCT), we performed a retrospective analysis of 66 Jehovah's Witnesses patients who underwent BL-ASCT and 1114 non-Jehovah's Witness patients who underwent transfusion-supported ASCT (TF-ASCT) at Cedars-Sinai Medical Center between January 2000 and September 2018. Survival was compared between the two groups. Transplant-related complications, mortality, engraftment time, length of hospital stay, and number of ICU transfers were characterized for the BL-ASCT group. One year survival was found to be $87.9 \%$ for both groups $(P=0.92)$. In the BL-ASCT group, there was one death prior to the 30 days post transplant due to CNS hemorrhage, and one death prior to 100 days due to sepsis. Based on our data, BL-ASCT can be safely performed with appropriate supportive measures, and we encourage community oncologists to promptly refer JW patients for transplant evaluation when ASCT is indicated.
\end{abstract}

These authors contributed equally: Alyssa Beck, Robert Lin, Ali Reza Rejali, Yuliya P. Linhares

Deceased: Michael Lill

Yuliya P. Linhares

Yuliyalin@gmail.com

1 Department of Internal Medicine, Cedars-Sinai Medical Center, 8700 Beverly Boulevard, Los Angeles, CA 90048, USA

2 Department of Pharmacy, Cedars-Sinai Medical Center, 8700 Beverly Boulevard, Los Angeles, CA 90048, USA

3 Miami Cancer Institute, Baptist Health South Florida, 8900 North Kendall Drive, Miami, FL 33176, USA

4 Department of Hematology/Oncology, Cedars-Sinai Medical Center, Samuel Oschin Comprehensive Cancer Institute, 8700 Beverly Boulevard, Los Angeles, CA 90048, USA

\section{Introduction}

High-dose chemotherapy (HDT) followed by autologous stem cell transplantation (ASCT) is the standard of care for patients newly diagnosed with multiple myeloma due to the improvement in progression-free survival (PFS) with this approach [1, 2]. ASCT for multiple myeloma patients beyond first remission may offer a survival benefit [3-5]. HDT followed by ASCT is a standard of care for relapsed diffuse large B cell lymphoma as well as multiple other lymphoma subtypes in different stages of treatment $[1,6]$. In the course of ASCT it is customary to provide red cell and platelet transfusion support on a preventative basis. Most institutions recommend red cell transfusion for hemoglobin levels $<7 \mathrm{~g} / \mathrm{dL}$, and platelet transfusion for platelet counts $<10 \times 10^{9} / \mathrm{L}$. On average, an ASCT recipient receives 1-2 units of PRBCs and 2 units of platelet transfusions in the posttransplant course [7,8]. Platelet and red cell posttransplant transfusion requirement may depend on institutional protocol, pretransplant hemoglobin and platelet 
counts $[9,10]$. Thus, given that ASCT is the standard of therapy for several malignancies, and given that it typically involves blood product transfusions, difficulties arise when a patient refuses blood transfusions.

In particular, the Jehovah's Witness (JW) faith is one of the more well-known religious groups which decline blood transfusions. The JW organization, the Watchtower Society, mandated the policy on refusal of blood transfusion in 1945 based on the following quote in the Bible, Leviticus 17:12 "None of you may eat blood, nor may any foreigner living among you eat blood" [11]. The ones that do choose to accept blood and platelet transfusions may be excluded from their religious community based on the following Biblical quote, Leviticus 17:10, "If anyone from the house of Israel or foreigner living among them eats any blood, I will set My face against that person and cut him off from among his people." JW who choose to accept blood may be subject to "disfellowship" from the JW organization as well as shunning and even isolation from their family and friends. Repentance and humility to the degree satisfactory to the JW elders may lead to the member "reinstatement" [12]. Due to these beliefs, JW patients are placed in a difficult position where they must choose to either receive standard medical care and get cast out from their religious community or choose to receive suboptimal care and remain within their religious community.

In addition, many JW patients with myeloma/lymphoma/leukemia had to forego ASCT when it is indicated due to the fact that most transplant centers do not offer stem cell transplant treatment without transfusion support. This may be due to perceived risks of increased risk of adverse events, such as bleeding and cardiovascular complications, and/or due to the lack of experience with bloodless transplants. Nevertheless, there is now a growing body of evidence that with appropriate supportive care, bloodless autologous stem cell transplants (BL-ASCT) can be as safe and effective as ASCT with transfusion support (TS-ASCT) [7, 8, 13, 14]. In this study we describe our center's experience with 70 BL-ASCT cases with regards to their engraftment outcomes, complications, mortality, and survival data.

\section{Materials and methods}

We reviewed electronic medical records of all patients who underwent BL-ASCT at our institution between January 2000 and September 2018. In order to obtain the comparator data for the OS, we reviewed contemporaneously matched medical records of the patients who underwent TSASCT for the same malignancy indications during the same time period. A total of 1180 patients, 66 (5.6\%) undergoing
BL-ASCT and 1114 (94.4\%) undergoing TS-ASCT were included in the study. The study was approved by the Institutional Review Board of the Cedars-Sinai Medical Center, Los Angeles, USA.

Patients with a malignancy indication for ASCT and adequate organ function were selected for autologous transplantation. Refusal of platelet and red cell transfusions did not exclude patients from ASCT. Candidates for BLASCT did not have to meet additional stem cell transplant eligibility criteria compared with the patients undergoing TS-ASCT. It was preferable for the patients to have pretransplant hemoglobin of $10 \mathrm{~g} / \mathrm{dL}$ and platelet count of equal or over $100 \times 10^{9} / \mathrm{L}$. There was no standardized protocol for the achievement of the desired hemoglobin and platelet thresholds pre-BL-ASCT.

Patients who met institutional criteria for ASCT underwent standardized granulocyte colony-stimulating factor (G-CSF) mobilization and apheresis for collection of stem cells. Patients were admitted prior to stem cell transplantation and given one of several conditioning regimens, including: melphalan, BEAM, R-BEAM, carboplatin + etoposide, carboplatin + etoposide + thiotepa, total body irradiation + cyclophosphamide, total body irradiation + cyclophosphamide + etoposide, busulfan + cyclophosphamide, carmustine + thiotepa.

Autologous stem cells were infused on day 0. During their hospital stay, all patients received standard prophylactic acyclovir/valacyclovir, levofloxacin,"-azole" antifungals, in addition to G-CSF (starting day +5 ).

\section{Supportive care}

A variety of measures were taken to minimize blood loss, decrease bleeding risk, and stimulate hematopoiesis for all patients (Table 1). These interventions include but are not limited to minimizing blood loss via collection of routine labs every other day and pediatric tube use, intravenous or oral iron supplementation, vitamin $\mathrm{K}$ supplementation, folic acid supplementation, erythropoietin, antiemetics, proton pump inhibitors, stool softeners, nasal saline spray, aminocaproic acid, strict bed rest under certain conditions, and (for female patients) oral contraceptives.

\section{Statistical analysis}

Neutrophil engraftment was defined as the first day of absolute neutrophil count $\geq 0.5 \times 10^{9} / \mathrm{L}$ for 3 consecutive days. Platelet engraftment is the first day of platelets $\geq 20 \times$ $10^{9} / \mathrm{L}$ for 3 consecutive days. Days were calculated with respect to day of hematopoietic stem cell infusion, which is designated as day 0. Patients who passed away due to transplantation-related mortality (TRM) before engraftment were censored at the date of their death. Posttransplant overall survival (OS) was defined from the date of 
Table 1 Supportive care measures for Jehovah's Witnesses undergoing HSCT.
Universal measures

Minimize phlebotomy (draw labs every other day in pediatric tubes, avoid unnecessary tests)

Vitamin K 10 mg PO TIW, folic acid $1 \mathrm{mg}$ PO TID, vitamin B12 $1 \mathrm{~g}$ PO daily

Stool softeners (e.g. docusate $250 \mathrm{mg}$ PO BID)

Aggressive antiemetic pharmacotherapy

Proton pump inhibitor (e.g., nexium $40 \mathrm{mg}$ PO daily)

Nasal saline spray

Ferrous sulfate 325 PO TID with ascorbic acid $100 \mathrm{mg}$ PO TID ${ }^{\mathrm{a}}$

Fall and bleeding precautions

Cessation of menses (e.g. medroxyprogesterone $10 \mathrm{mg}$ daily)

No NSAIDs or aspirin

G-CSF following completion of chemotherapy and transplant

Aggressive treatment of fever with acetaminophen

Avoid myelosuppressive antibiotics (e.g., bactrim, linezolid)

Erythropoietin 150 units/kg SQ TIW starting Day +1 to keep $\mathrm{Hgb}>11 \mathrm{~g} / \mathrm{dL}$. May increase to 150 units/kg SQ daily

\section{Anemia-Hgb less than}

$10 \mathrm{~g} / \mathrm{dL} \quad$ Iron sucrose $100 \mathrm{mg}$ IV weekly for 4 weeks (if not already on and in place of oral iron)

$6 \mathrm{~g} / \mathrm{dL} \quad$ Bed rest

Oxygen $2 \mathrm{~L} / \mathrm{min}$ via nasal cannula

Bedside commode with assistance

Vitals q4 h

Initiate "fall precautions" including bed alarm

$4 \mathrm{~g} / \mathrm{dL} \quad$ Absolute bed rest

Bedpan and urinal in bed only (no commode or bathroom use)

Oxygen via nonrebreather at all times

Minimize coughing, retching, vomiting via optimal medical management

\section{Thrombocytopenia-platelets less than}

$50 \times 10^{9} / \mathrm{L}$ with Start aminocaproic acid $1-4 \mathrm{~g}$ PO/IVPB q4-6 h, adjust dose until bleeding resolves bleeding DDAVP $0.3 \mathrm{mcg} / \mathrm{kg}$ IVPB $\mathrm{q} 12 \mathrm{~h}$ for $36 \mathrm{~h}$ if bleeding persists on aminocaproic acid Nasal vasoconstrictors for epistaxis (e.g., neosynephrine)

Conjugated estrogens PRN for vaginal bleeding (e.g., Premarin $25 \mathrm{mg}$ IVP)

$10 \times 10^{9} / \mathrm{L}$

Start low-dose aminocaproic acid 1-4 g PO/IVPB q4-6h

Obtain urinalysis prior to starting aminocaproic acid (assess for hematuria)

Vitamin K 5-10 mg IVPB daily

Stop all anticoagulation

$5 \times 10^{9} / \mathrm{L} \quad$ Topical aminocaproic acid if patient has mucositis

$H S C T$ hematopoietic stem cell transplant, TIW three times weekly, DDAVP desmopressin, $G$-CSF granulocyte colony-stimulating factor (e.g., filgrastim), NSAID nonsteroidal anti-inflammatory drug

${ }^{a}$ Changed in June 2013 to iron sucrose $100 \mathrm{mg}$ IV weekly as a universal measure regardless of $\mathrm{Hgb}$ to avoid risk of GI bleed with oral iron supplementation transplantation to the date of death or last follow-up. OS was estimated using the Kaplan-Meier method and was compared using the log-rank test.

Kaplan-Meier was also used to determine median follow-up post transplant. Nominal data were compared using Chi-square or Fisher exact test, while ordinal data were compared using the Mann-Whitney test. All statistical analyses were performed using GraphPad Prism version 8.1.2 for Windows, GraphPad Software, La Jolla California USA, www.graphpad.com.

We performed a limited analysis of the TS-ASCT data for the purposes of comparing OS between the BL-ASCT and TS-ASCT patients. We included only first transplant events for the OS calculations. 
Table 2 Patient characteristics.

\begin{tabular}{|c|c|c|c|}
\hline & BL-ASCT & TS-ASCT & $p$ \\
\hline Total number of patients & 66 & 1114 & \\
\hline Total number of transplants & 70 & 1212 & \\
\hline \multicolumn{4}{|l|}{ Age at 1 st transplant, years } \\
\hline Median & 53 & 56 & \multirow[t]{2}{*}{0.03} \\
\hline Range & $17-72$ & $17-84$ & \\
\hline \multicolumn{4}{|l|}{ Sex } \\
\hline Male & $31(47 \%)$ & $663(60 \%)$ & \multirow[t]{2}{*}{0.04} \\
\hline Female & $35(53 \%)$ & $451(40 \%)$ & \\
\hline \multicolumn{4}{|l|}{ Ethnicity } \\
\hline Caucasian & $24(36 \%)$ & $686(62 \%)$ & \multirow[t]{5}{*}{0.12} \\
\hline Hispanic & $22(33 \%)$ & $221(20 \%)$ & \\
\hline African American & $15(23 \%)$ & $70(6 \%)$ & \\
\hline Asian & $4(6 \%)$ & $99(9 \%)$ & \\
\hline Other & $1(2 \%)$ & $38(3 \%)$ & \\
\hline \multicolumn{4}{|l|}{ Diagnosis } \\
\hline Multiple myeloma & $31(47 \%)$ & $646(58 \%)$ & \multirow[t]{6}{*}{0.12} \\
\hline Non-Hodgkin lymphoma & $17(26 \%)$ & $310(28 \%)$ & \\
\hline Hodgkin lymphoma & $13(19 \%)$ & $106(10 \%)$ & \\
\hline Testicular germ cell tumor & $2(3 \%)$ & $25(2 \%)$ & \\
\hline Acute myelogenous leukemia & $1(2 \%)$ & $27(2 \%)$ & \\
\hline Acute lymphoblastic leukemia & $2(3 \%)$ & 0 & \\
\hline \multicolumn{4}{|c|}{ Median follow-up post transplant, months } \\
\hline After 1st AUTO SCT & 63 & 90 & 0.03 \\
\hline
\end{tabular}

\section{Results}

\section{Patient characteristics}

Patient characteristics are summarized in Table 2. There were 70 BL-ASCT transplants performed from June 2000 to December 2018. Diagnoses included multiple myeloma $(n=31)$, Non-Hodgkin lymphoma $(n=17)$, Hodgkin lymphoma $(n=13)$, ALL $(n=2)$, AML $(n=1)$, and germ cell tumor $(n=2)$. Four of the seventy transplant cases were second BL-ASCT cases with the first BL-ASCT performed at Cedars-Sinai and included in this analysis. A total of 66 patients received BL-ASCT. The median age at the time of first transplantation was 53 years (range 17-72 years old) in the BL-ASCT.

We reviewed select data for TS-ASCTs performed from January 2000 to December 2018. We identified 1114 patients who underwent TS-ASCT for the indications matching BL-ASCT (multiple myeloma, lymphoma, germ cell tumor, acute lymphoblastic leukemia, or acute myeloid leukemia). Patients who underwent TS-ASCT were older than BL-ASCT patients with a median age of 56 years (range 17-84) at the time of first transplant.
Table 3 Engraftment parameters for BL-ASCT patients.

\begin{tabular}{llll}
\hline & Median & Mean & Range \\
\hline CD34 $\times 10^{6} / \mathrm{kg}$ infused & 6.4 & 7.1 & $1.1-27.5$ \\
Pretransplant $\mathrm{Hgb}(\mathrm{g} / \mathrm{dL})$ & 12.6 & 12.6 & $9.4-15.4$ \\
Days to Hgb nadir & 11 & 11.5 & $1-28$ \\
Days Hgb below $7 \mathrm{~g} / \mathrm{dL}$ & 0 & 0.6 & $0-14$ \\
Mean Hgb $(\mathrm{g} / \mathrm{dL})$ & 10.5 & 10.6 & $7.6-14.8$ \\
Hgb nadir $(\mathrm{g} / \mathrm{dL})$ & 8.5 & 8.7 & $5.4-14$ \\
Pretransplant PL $\left(\times 10^{9} / \mathrm{L}\right)$ & 189 & 200 & $71-478$ \\
Days to PL nadir & 9 & 9 & $5-21$ \\
Days PL below $10 \times 10^{9} / \mathrm{L}$ & 3 & 3.7 & $0-17$ \\
Mean PL $\left(\times 10^{9} / \mathrm{L}\right)$ & 91 & 93 & $31-163$ \\
PL nadir $\left(\times 10^{9} / \mathrm{L}\right)$ & 4.5 & 6.5 & $1-38$ \\
Day $+30 \mathrm{Hgb}(\mathrm{g} / \mathrm{dL})$ & 11.6 & 11.4 & $5.9-15$ \\
Day $+30 \mathrm{PL}\left(\times 10^{9} / \mathrm{L}\right)$ & 208 & 202 & $5-554$ \\
Time to NF engraftment (days) & 12 & 11.9 & $9-17$ \\
Time to PL engraftment (days) & 13 & 14.5 & $9-38$ \\
Duration of grade 3-4 anemia (days) & 0 & 1.8 & $0-19$ \\
Duration of Gr 3 TCP (days) & 9 & 10.1 & $0-34$ \\
Duration of Gr 4 TCP $($ days $)$ & 5 & 6.5 & $0-29$ \\
\hline$P L$ ) & 5 & &
\end{tabular}

$P L$ platelet, $N F$ neutrophil, $T C P$ thrombocytopenia

\section{BL-ASCT engraftment data}

According to the institutional standard operating procedure, the requested stem cell dose for one ASCT was at least $5 \times$ $10^{6} \mathrm{CD} 34$ cells $/ \mathrm{kg}$. A median of $6.4 \times 10^{6} \mathrm{CD} 34+/ \mathrm{kg}$ cells were infused (range 1.1-27.5 × $10^{6} \mathrm{CD} 34$ cells/kg). Few patients were unable to achieve the target collection, but were transplanted after the risk versus benefit discussion. Engraftment data are reported in Table 3. The average hemoglobin prior to conditioning therapy was $12.6 \mathrm{~g} / \mathrm{dL}$ (range 9.4-15.4 g/dL). We evaluated hemoglobin, platelets and neutrophil engraftment up to day +30 post auto SCT. When the blood count data were not available on day +30 , we used the blood count data from the day closest to day +30 (mean and median day +28 , range 14-41 days). Patients experienced a median decrease in hemoglobin of $4.1 \mathrm{~g} / \mathrm{dL}$ (range $1.4-4 \mathrm{~g} / \mathrm{dL}$ ). The median number of days with hemoglobin $<7 \mathrm{~g} / \mathrm{dL}$ was 0 (mean 0.6 , range $0-14$ ). The median day +30 hemoglobin was $11.6 \mathrm{~g} / \mathrm{dL}$.

There was platelet decrease in all patients. Average platelet count prior to conditioning therapy was $199.7 \times 10^{9} / \mathrm{L}$ (range $71-478 \times 10^{9} / \mathrm{L}$ ). We evaluated platelet counts up to day +30 post-SCT. The median decrease in platelets was $185 \times 10^{9} / \mathrm{L}$ (range $70-440 \times 10^{9} / \mathrm{L}$ ). Median platelet count was $91 \times 10^{9} / \mathrm{L}$ (range $\left.31-163 \times 10^{9} / \mathrm{L}\right)$. Median platelet nadir was $4.5 \times 10^{9} / \mathrm{L}$ (range $1-38 \times 10^{9} / \mathrm{L}$ ). The median days to platelet nadir was 9 days (range 5-21 days). Patients experienced platelet engraftment after a median of 13 days (range 9-38 days). On day +30 , the median platelet count 
was $208 \times 10^{9} / \mathrm{L}$ (range $5-554 \times 10^{9} / \mathrm{L}$ ). CTCAE 5.0 Grade 4 thrombocytopenia lasted a median of 5 days (range 0-29 days). The median number of days with platelet count $<10 \times 10^{9} / \mathrm{L}$ was 3 (range $0-17 \times 10^{9} / \mathrm{L}$ ). One patient with AML engrafted neutrophils, but failed to engraft platelets by day +21 and was given a second stem cell infusion on day +24 with subsequent platelet engraftment on day +38 . The only patients who did not engraft platelets were the two patients who experienced TRM prior to day +100 .

All patients, except for the two patients who died prior to day +100 experienced neutrophil engraftment. The average number of days to neutrophil engraftment was 12 (range 9-17 days).

\section{BL-ASCT cardiovascular complications}

There were six cardiac complication events $(8.6 \%)$ with three patients being transferred to ICU. No fatalities resulted from cardiovascular complications. Four patients experienced atrial fibrillation and two had episodes of SVT requiring adenosine without subsequent events. None of the patients experienced bradycardia or hypotension due to a cardiac event. None of the patients who experienced cardiac events had hemoglobin nadir below $7 \mathrm{~g} / \mathrm{dL}$. BL-ASCT mean hemoglobin nadir was identical at $8.7 \mathrm{~g} / \mathrm{dL}$ in patients who experienced cardiac complications and those who did not. Hemoglobin range was $11.6-7.5 \mathrm{gm} / \mathrm{dL}$ in BL-ASCT patients who experienced cardiac complications. Hemoglobin range was $14-5.4 \mathrm{~g} / \mathrm{dL}$ in BL-ASCT patients who did not experience cardiac complications.

\section{BL-ASCT bleeding complications}

Bleeding complications were classified using the WHO grading system and summarized in Table 4 . There were 12 bleeding episodes $(17.1 \%)$.

There was one fatal bleeding complication secondary to a CNS hemorrhage in a 30-year-old female patient with central nervous system germ cell tumor with persistent disease in the pineal gland area prior to transplantation. CNS hemorrhage happened on day +3 post-BL-ASCT with the platelet nadir of $31 \times 10^{9} / \mathrm{L}$ (average $91 \times 10^{9} / \mathrm{L}$ ). There were eight episodes $(11.4 \%)$ of WHO grade $1-2$ bleeding, including two genitourinary, two hematemesis, three epistaxis one of which co-existed with vaginal bleeding, and two mucocutaneous. These episodes did not warrant ICU transfers and resolved with appropriate medical management. There were two grade 3 lower GI bleeding episodes (2.9\%), and one grade 3 vaginal/epistaxis bleeding episode, neither required ICU transfer due to the bleeding event. Of the 12 patients who experienced bleeding events of any grade, three patients had hemoglobin decline to below $7 \mathrm{~g} / \mathrm{dL}$, none had hemoglobin decline to below $5 \mathrm{~g} / \mathrm{dL}$. In 9 out of 12 patients with bleeding events (75\%), platelet nadir was below $5 \times 10^{9} / \mathrm{L}$.

\section{Neurologic complications}

There were no fatalities resulting from neurologic complications. One patient had a tonic-clonic seizure without any subsequent episodes. The patient did not require an ICU transfer.

\section{Hospitalization and ICU transfers for the BL-ASCT}

Hospitalization for the BL-ASCT lasted a mean of 18.5 days (range $11-36$ days). Out of 70 BL-ASCT events, there were six ICU transfers (11.7\%), three of which were due to cardiovascular complications and one due to CNS bleeding. Mean number of days spent in the ICU was 0.34 (range 0-13).

\section{Mortality and TRM}

There was 1 death out of 70 transplant events prior to day +30 . This was a patient with a history of CNS germ cell tumor with persistent disease in the pineal gland area prior to transplant who succumbed to intracranial hemorrhage which was considered TRM. The second death event before day 100 was in a patient with multiple myeloma who contracted H1N1 influenza before day +30 post-SCT, developed ARDS, sepsis and died of multiorgan failure despite maximum supportive care. This death was considered to be TRM. Subsequently, no deaths were considered TRM. Overall, 100 day mortality was $2.9 \%$ out of 70 transplant events. Among the additional six patients who died after day +100 , five patients died of disease progression, while one patient died of unknown cause close to 1 year post-SCT (cause unclear due to the lack of medical records). BL-ASCT TRM was $2.9 \%$. One year mortality for the whole BL-ASCT cohort was $11.4 \%$.

\section{Survival}

With a median follow-up of 63 months, 45 BL-ASCT patients are alive. The posttransplant OS was $87.9 \%$ at 1 year for all BL-ASCT cases. There was no mortality prior to 1 year post transplant for those who received their second BL-ASCT. 30 day, 100 day, 1 year, and 5 year posttransplant OS did not differ between the BL-ASCT and TSASCT groups as illustrated in Fig. 1. Median OS was not reached for either group with a median follow-up of 63 months in the BL-ASCT group and 90 months for the TS-ASCT group.

Of the 70 BL-ASCT transplant cases, four cases are second transplants for the patients who had first BL-ASCT 
Table 4 Post transplant complications in BL-ASCT group.

\begin{tabular}{|c|c|c|c|c|c|c|c|}
\hline Age gender & Diagnosis & $\begin{array}{l}\text { Conditioning } \\
\text { regimen }\end{array}$ & Days in ICU & $\begin{array}{l}\text { Bleeding } \\
\text { WHO grade }\end{array}$ & $\begin{array}{l}\text { PL Nadir } \times \\
10^{9} / \mathrm{L}\end{array}$ & $\begin{array}{l}\mathrm{Hgb} \\
\text { Nadir g/dL }\end{array}$ & Comments \\
\hline $5 \mathrm{~F}$ & MM & Mel200 & 0 & $\leq 2$ & 38 & 12.8 & Mild hematemesis \\
\hline $62 \mathrm{~F}$ & NHL & R-BEAM & 0 & $\leq 2$ & 2 & 8.3 & Hematemesis \\
\hline $57 \mathrm{~F}$ & MM & Mel100 & 13 & 3 & 1 & 5.4 & $\begin{array}{l}\text { Cardiac tamponade, rectal bleeding, } \\
\text { H1N1 influenza, ARDS, sepsis, and } \\
\text { multiorgan failure }\end{array}$ \\
\hline $30 \mathrm{~F}$ & CNS GCT & Carbo/Thio/VP16 & 3 & 4 & 31 & 14 & $\begin{array}{l}\text { CNS bleed, died despite maximum } \\
\text { supportive care }\end{array}$ \\
\hline $21 \mathrm{M}$ & $\mathrm{HL}$ & BEAM & 0 & 3 & 1 & 6.6 & Lower GI bleeding \\
\hline $37 \mathrm{~F}$ & $\mathrm{HL}$ & BEAM & 0 & 3 & 5 & 6.4 & $\begin{array}{l}\text { Vaginal bleeding, epistaxis, blood } \\
\text { in sputum }\end{array}$ \\
\hline $64 \mathrm{M}$ & NHL & R-BEAM & 0 & $\leq 2$ & 3 & 7.1 & Hematuria \\
\hline $24 \mathrm{~F}$ & $\mathrm{HL}$ & BEAM & 0 & $\leq 2$ & 2 & 8.9 & Vaginal spotting \\
\hline $17 \mathrm{~F}$ & NHL & BEAM & 0 & $\leq 2$ & 5 & 7.3 & Gum bleeding \\
\hline $47 \mathrm{M}$ & NHL & BEAM & 0 & $\leq 2$ & 2 & 7.2 & Petechiae lower extremities \\
\hline $53 \mathrm{~F}$ & MM & Mel200 & 0 & $\leq 2$ & 2 & 7.7 & Mild epistaxis \\
\hline $52 \mathrm{~F}$ & MM & Mel200 & 0 & $\leq 2$ & 9 & 8.6 & Epistaxis \\
\hline $67 \mathrm{~F}$ & MM & Mel200 & 1 & & 2 & 8.3 & A fib \\
\hline $61 \mathrm{M}$ & MM & Mel200 & 2 & & 8 & 7.5 & A fib w/RVR \\
\hline $66 \mathrm{~F}$ & NHL & R-BEAM & 0 & & 2 & 8.1 & A fib w/RVR \\
\hline $60 \mathrm{M}$ & NHL & BEAM & 0 & & 3 & 7.7 & A fib \\
\hline $53 \mathrm{M}$ & MM & Mel200 & 0 & & 13 & 11.6 & SVT, adenosine administered \\
\hline $57 \mathrm{M}$ & MM & Mel200 & 4 & & 5 & 9 & SVT, HTN \\
\hline $61 \mathrm{~F}$ & ALL & $\mathrm{TBI} / \mathrm{Cy}$ & 0 & & 4 & 10.4 & Tonic-clonic seizure \\
\hline
\end{tabular}

$M M$ multiple myeloma, $N H L$ non-Hodgkin's lymphoma, $H L$ Hodgkin's lymphoma, $C N S G C T$ central nervous system germ cell tumor, $A L L$ acute lymphoblastic lymphoma, Mel melphalan, Carbo carboplatin, Thio thiotepa, VP16 etoposide, $R$ rituximab, ARDS acute respiratory distress syndrome, GI gastrointestinal, A fib atrial fibrillation, RVR rapid ventricular response, SVT supraventricular tachycardia, HTN hypertension

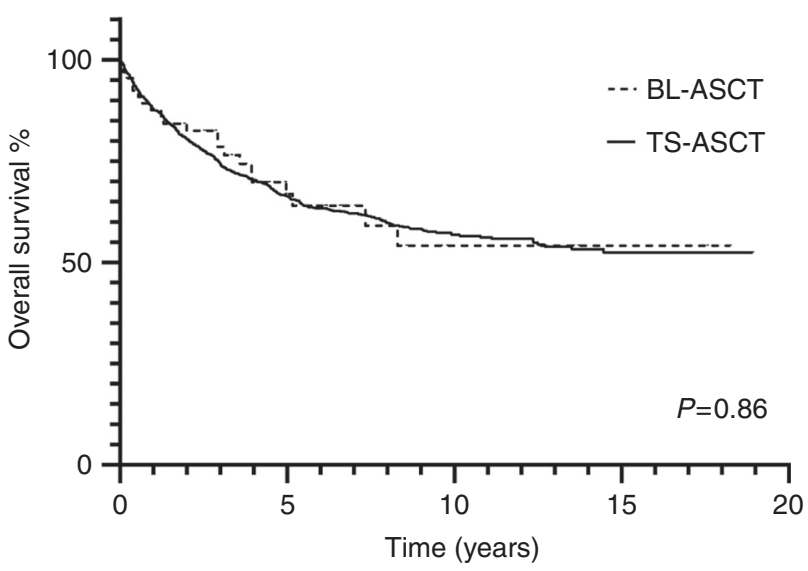

Fig. 1 BL-ASCT and TS-ASCT patient Kaplan-Meier survival curve.

at our institution. None of the second transplant cases experienced mortality prior to 1 year post transplant. Out of 70, 68 BL-ASCT cases were alive at 100 days $(97 \%)$ postSCT and 62 out of 70 BL-ASCT cases were alive at 1 year $(88.6 \%)$ post-SCT.

In addition, posttransplant survival between BL-AST and TS-AST, stratified by subgroups, such as age categories
( $<40$ years, 40-59 years and $\geq 60$ years), sex (male and female), ethnicity (Caucasian, Hispanic and African American), and diagnosis (multiple myeloma, non-Hodgkin's lymphoma, Hodgkin lymphoma, and acute leukemia), did not show any significant difference.

\section{Discussion}

ASCT improved OS and PFS in multiple malignancies, including relapsed lymphomas and multiple myeloma, and is a standard of care for the treatment of relapsed aggressive B cell lymphomas and Hodgkin lymphoma. Very few transplant centers in the United States and world-wide offer BL-ASCT.

This study supports the concept that BL-ASCT can be performed safely without significantly increased risk of TRM. In our study, there were only 2 cases of TRM out of 70 transplant cases with overall BL-ASCT TRM at 2.9\%, which is $<8 \%$ TRM in bloodless transplants reported by Ballen et al. and comparable with $2-4 \%$ TRM in TS-ASCT $[2,7,14,15]$. Joseph et al. reported the lowest $0 \%$ TRM in BL-ASCT for multiple myeloma [7]. 
Supraventricular arrhythmia is a common complication of traditional autologous SCT. In 6 out of $70(8.6 \%)$ BLASCT transplants, patients experienced cardiac complications manifested as arrhythmias. We do not believe there was a clinically significant increase in the incidence of arrhythmias in BL-ASCT cases as compared with previously described arrhythmia incidence in TS-ASCT. Analysis of 983 TS-ASCT cases in Nebraska demonstrated 9.4\% incidence of arrhythmias [16]. In our study, arrhythmias were not associated with the degree of anemia in BLASCT patients, as the mean nadir hemoglobin nadir was identical at $8.7 \mathrm{~g} / \mathrm{dL}$ in patients who experienced arrhythmias and those who did not. Based on our data, it does not appear that the arrhythmic complications in BL-ASCT group were related to the degree of anemia.

A platelet level of $<10 \times 10^{9} / \mathrm{L}$ makes bleeding one of the most feared complications in BL-ASCT $[13,14]$. In our study, there were four episodes of bleeding in 70 transplant cases with a WHO grade $>2$ and eight episodes of WHO grade 1-2 bleeding. In most cases, bleeding resolved promptly with medical management. Only one of the four higher grade bleeding patients had to be transferred to ICU due to hemorrhage. The second patient with higher grade bleeding had multiple medical issues requiring ICU transfer. Joseph et al. reported no bleeding-associated mortality in their series of 24 BL-ASCT cases, while Ballen et al. reports two cases of high-grade bleeding in 26 BL-ASCT patients [7, 14]. Based on our data, bleeding risk appears to be clinically acceptable to perform BL-ASCT transplants. In our study, most bleeding episodes happened during the time when the patients had platelet nadir below $5 \times 10^{9} / \mathrm{L}$, which is consistent with previously reported data by Ford et al. where no bleeding episodes occurred in BL-ASCT patients with platelets $>5 \times 10^{9} / \mathrm{L}$ [13]. It is important to point out that at our institution as well as in all published BL-ASCT protocols, aminocaproic acid was administered to patients undergoing BL-ASCT when the platelet level decreased below $10 \times 10^{9} / \mathrm{L}$. Platelet threshold for the initiation of aminocaproic acid as well as aminocaproic acid dose vary between institutions $[7,8,10,13,14]$. There are no studies comparing the incidence and severity of bleeding with or without aminocaproic acid in the setting of BL-ASCT. In a prospective study, Wandt et al. demonstrated that TS-ASCT patients can be managed safely with therapeutic platelet transfusions without routine aminocaproic acid utilization [17]. Similarly, a randomized, open-label multicenter trial of prophylactic versus therapeutic platelet transfusions in patients with hematologic cancers demonstrated similar rates of bleeding in the two study groups among ASCT patients, and aminocaproic acid prophylaxis was not routinely used [18]. We support the updated 2017 ASCO guideline for the TS-ASCT which, allows experienced centers to use therapeutic platelet transfusion with the first sign of bleeding in adult patients as the majority of transplant-related bleeding episodes are nonlife threatening and resolve with supportive care even with the platelet transfusion-free approach [19]. The use of aminocaproic acid in therapeutic platelet TS-ASCT remains upon the discretion of the treating physician.

To date, there is no sufficient evidence to support the use of thrombopoietin mimetics in conventional or BL-ASCT. Several studies including our analysis report no bleedingassociated mortality in BL-ASCT performed without routine or emergent use of thrombopoietin mimetics [7, 15]. Coltoff et al. administered romiplostim prior to BL-ASCT to two patients who subsequently did not meet indications for transfusions and did not experience any bleeding. AlNawakil et al. administered romiplostim to 10 out of 13 patients on BL-ASCT protocol with mean duration of thrombocytopenia of $<10 \times 10^{9} / \mathrm{L}$ at 4.5 days [20]. In contrast, in our study, the median number of days with platelet count $<10 \times 10^{9} / \mathrm{L}$ was 3 without thrombopoietin mimetic use. Based on the current safety profile of BL-ASCT and due to the paucity of data regarding thrombopoietin mimetic use in ASCT we do not support routine use of thrombopoietin mimetics in conventional or BL-ASCT.

The utility and feasibility of red blood cell substitutes is a topic frequently addressed by patients who do not accept blood transfusions and their physicians. Unfortunately, none of the blood substitutes are approved by the US Food and Drug Administration. Synthetic oxygen transporters currently in development include perfluorocarbons and human or bovine hemoglobin oxygen carriers (HBOC). HBOCs can temporarily supply hemoglobin for oxygen delivery in critical patients with profound but reversible anemia. Hemoglobin based Oxygen Carrier HBOC-201 (Hemopure) is available for compassionate use for lifethreatening anemia for patients where allogeneic blood transfusion is not an option. There are multiple case reports describing HBOC-201 use in JWs with critical anemia due to trauma, surgical complications, or acute medical illness. HBOC consistently provides increases in hemoglobin allowing time to perform additional measures for patient stabilization [20-23]. There are no reports of HBOC or perfluorocarbon use in stem cell transplantation. Compassionate use of HBOC-201 should be considered for critically ill JW with severe transient anemia undergoing stem cell transplantation where a temporary increase in tissue oxygen delivery would provide opportunity for other lifesaving measures.

In our series, the patient with a history of CNS germ cell tumor with persistent disease in the pineal gland area prior to BL-ASCT succumbed to an intracranial hemorrhage early post-BL-ASCT. Ballen et al. report death from the intracranial bleed in a patient with active medulloblastoma [14]. We suggest exercising caution in performing 
BL-ASCT in patients with active CNS malignancy or a history of solid CNS malignancy.

In our study, OS did not differ between the patients who underwent BL-ASCT and the patients who underwent TSASCT for the same indications. Joseph et al. from the Emory University reported 24 multiple myeloma patients who underwent BL-ASCT with OS comparable with the matched cohort of TS-ASCT [7]. Therefore, all appropriate ASCT candidates who do not accept blood transfusions should have access to BL-ASCT.

The length of stay for the BL-ASCT as well as TS-ASCT varies between the studies. The median length of stay for the BL-ASCT patients in our study was 18 days (range 11-36 days), which is longer than 15 days (range 12-24 days) in BL-ASCT for the myeloma indication reported by Joseph et al., but comparable with 19 days (range 5-35 days) in BL-ASCT reported by Ford et al. For the TS-ASCT, Ford et al. reported average length of stay of 17 days (range 6-76 days) and Joseph et al. reported 16 days (range 12-29) [7, 13]. Based on these data, transplant centers should not expect clinically significant increased length of stay for BL-ASCT cases.

A limitation of this study is that this is a retrospective chart review and certain data points, such as disease status, could not be assessed to report PFS. The supportive care measures for the BL-ASCT were chosen empirically based on the theoretical benefit and were not studied in randomized controlled trials.

The Pew Research Center reports that JWs make up $0.8 \%$ of the US population at an $\sim 2.5$ million people, making an encounter with JW patients who need ASCT a common occurrence [24]. In our experience, JW patient referral to autologous transplant is frequently delayed as there is no widespread knowledge in the community and university practices that ASCT can be performed safely without significant increase in TRM in appropriate candidates. JWs have to travel long distances in order to obtain ASCT consultation and frequently have to undergo BLASCT far from their home. Complicated logistics of the BL-ASCT increases administrative burden on the referring oncologists and transplant institutions and causes financial and emotional stress to the transplant patients. Based on our data, we encourage community oncologists to promptly refer JW patients for transplant evaluation when ASCT is indicated. We encourage all transplant centers to embark on starting BL-ASCT programs.

Acknowledgements We would like to acknowledge that ML who developed the Bloodless Stem Cell Transplant Program at Cedars-Sinai Medical Center passed away in June 19, 2018. We would like to thank the Honest EIS Research Broker committee for their contribution in data abstraction. We thank Teresa Devera for her assistance coordinating the financial aspects of patient care. We would like to show gratitude to social worker Julia Leavitt and administrative assistant Diana Rivera.
The publication of this article is generously supported by Soraya Ruben Melamed and the Melamed family of Beverly Hills.

\section{Compliance with ethical standards}

Conflict of interest The authors declare that they have no conflict of interest.

Publisher's note Springer Nature remains neutral with regard to jurisdictional claims in published maps and institutional affiliations.

Open Access This article is licensed under a Creative Commons Attribution 4.0 International License, which permits use, sharing, adaptation, distribution and reproduction in any medium or format, as long as you give appropriate credit to the original author(s) and the source, provide a link to the Creative Commons license, and indicate if changes were made. The images or other third party material in this article are included in the article's Creative Commons license, unless indicated otherwise in a credit line to the material. If material is not included in the article's Creative Commons license and your intended use is not permitted by statutory regulation or exceeds the permitted use, you will need to obtain permission directly from the copyright holder. To view a copy of this license, visit http://creativecommons. org/licenses/by/4.0/.

\section{References}

1. National Comprehensive Cancer Network Guidelines in Oncology. B Cell Lymphomas (version 2.2018). 2019. https://www. nccn.org/professionals/physician_gls/pdf/b-cell.pdf. Accessed 13 Nov 2019.

2. Attal M, Lauwers-Cances V, Hulin C, Leleu X, Caillot D, Moreau P. Lenalidomide, bortezomib, and dexamethasone with transplantation for myeloma. N. Engl J Med. 2017;376:1311-20.

3. Fermand JP, Ravaud P, Chevret S, Divine M, Leblond V, Belanger $\mathrm{C}$, et al. High-dose therapy and autologous peripheral blood stem cell transplantation in multiple myeloma: up-front or rescue treatment? Results of a multicenter sequential randomized clinical trial. Blood. 1998;92:3131-6.

4. Shah N, Ahmed F, Bashir Q, Qureshi S, Dinh Y, Rondon G, et al. Durable remission with salvage second auto transplants in patients with multiple myeloma. Cancer. 2012;118:3549-55.

5. Jimenez-Zepeda VH, Mikhael J, Winter A, Franke N, Masih-Khan E, Reece DE, et al. Second autologous stem cell transplantation as salvage therapy for multiple myeloma: impact on progression-free and overall survival. Biol Blood Marrow Transplant. 2012;18:773-9.

6. Gisselbrecht C, Glass B, Mounier N, Gill D, Linch D, Trneny M, et al. Salvage regimens with autologous transplantation for relapsed large B-cell lymphoma in the rituximab era. Clin Oncol. 2010;28:4184-90.

7. Joseph NS, Kaufman JL, Boise LH, Valla K, Almaula DK, Obidike $\mathrm{CO}$, et al. Safety and survival outcomes for bloodless transplantation in patients with myeloma. Cancer. 2019;125:185-93.

8. Khan R, Mott SL, Schultz A, Jethava YS, Tricot G. Bloodless tandem autologous transplant in Jehovah's Witness patients. Bone Marrow Transpl. 2018;53:1428-33.

9. Bolwell BJ, Goormastic M, Andresen S, Overoyer B, Pohlman B, Kalaycio M. Platelet transfusion requirements during autologous peripheral blood progenitor cell transplantation correlate with the pretransplant platelet count. Bone Marrow Transpl. 1997;20:459-63. 
10. Coltoff A, Shreenivas A, Afshar S, Steinberg A. A single-institution experience of performing bloodless transplant in Jehovah's Witness patients. Hematol Oncol Stem Cell Ther. 2019;12:44-9.

11. Muramoto O. Bioethical aspects of the recent changes in the policy of refusal of blood by Jehovah's Witnesses. Br Med J. 2001;322:37-9.

12. Lee E. Why some Jehovah's Witnesses accept blood and conscientiously reject official Watchtower Society blood policy. J Med Ethics. 2000;26:375-80.

13. Ford PA, Grant SJ, Mick R, Keck G. Autologous stem-cell transplantation without hematopoietic support for the treatment of hematologic malignancies in Jehovah's Witnesses. J Clin Oncol. 2015;33:1674-9.

14. Ballen KK, Becker PS, Yeap BY, Matthews B, Henry DH, Ford PA. Autologous stem-cell transplantation can be performed safely without the use of blood-product support. J Clin Oncol. 2004;22:4087-94.

15. Corradini P, Ladetto M, Zallio F, Astolfi M, Rizzo E, Sametti S, et al. Long-term follow-up of indolent lymphoma patients treated with high-dose sequential chemotherapy and autografting: evidence that durable molecular and clinical remission frequently can be attained only in follicular subtypes. J Clin Oncol. 2004;22:1460-8.

16. Singla A, Hogan WJ, Ansell SM, Buadi FK, Dingli D, Dispenzieri A, et al. Incidence of supraventricular arrhythmias during autologous peripheral blood stem cell transplantation. Biol Blood Marrow Transplant. 2013;19:1233-7.

17. Wandt H, Schaefer-Eckart K, Frank M, Birkmann J, Wilhelm M. A therapeutic platelet transfusion strategy is safe and feasible in patients after autologous peripheral blood stem cell transplantation. Bone Marrow Transpl. 2006;37:387-92.

18. Stanworth SJ, Estcourt LJ, Powter G, Kahan B, Dyer C, Choo L, et al. A no-prophylaxis platelet-transfusion strategy for hematologic cancers. N. Engl J Med. 2013;368:1771-80.

19. American Society of Clinical Oncology. ASCO Guidelines [Internet]. American Society of Clinical Oncology. 2017. Available from: https://www.asco.org/sites/new-www.asco.org/files/content-files/ 2017-platelet-transfusion-slides.pdf. Accessed 13 Nov 2019.

20. Al-Nawakil C, Quarre MC, Heshmati F, Deau B, Park S, Dreyfus $\mathrm{F}$, et al. Autologous stem cell transplantation in patients who object to a blood transfusion: contribution of new pharmacological haematopoiesis support. Br J Haematol. 2013;5:738-4020.

21. Moradi S, Jahanian-Najafabadi A, Roudkenar MH. Artificial blood substitutes: first steps on the long route to clinical utility. Clin Med Insights Blood Disord. 2016;9:33-41.

22. Bari S, Rabinovich M, Curry M, Jain SR, Cafuir L. Use of hemoglobin based oxygen carrier HBOC-021 (Hemopure) as a bridge during emergencies in patients unable to receive blood products: experience at a tertiary care center. Blood. 2017; 130(Suppl 1):4926.

23. Posluszny JA, Napolitano LM. Hemoglobin-based oxygen carrier for traumatic hemorrhagic shock treatment in a Jehovah's Witness. Arch Trauma Res. 2016;5:e30610.

24. Pew Research Center. Religious Landscape Study [Internet]. Lipka M. A closer look at Jehovah's Witnesses living in the U.S. April 26, 2016. Available from: https://www.pewresearch.org/facttank/2016/04/26/a-closer-look-at-jehovahs-witnesses-living-in-theu-s/. Accessed 13 Nov 2019. 